Editorial

\title{
Zika virus infection and disease
}

Keywords: Zika virus, Infection, Disease, Flaviviruses, Symptoms

\section{Editorial}

Zika virus is a disease transmitted by mosquitoes, particularly the Aedes aegypti. Aedes albopictus mosquitoes also might transmit the virus. Zika virus is a member of the Flaviviridae family and is related to other pathogenic vector-borne flaviviruses including dengue, Japanese encephalitis, and West-Nile viruses, but produces a comparatively mild clinical manifestations in humans. There is no indication that it can spread person to person through casual contact. Nevertheless, the United States Centers for Disease Control and Prevention has confirmed the first American case of Zika virus infection in a non-traveler in the continental United States after the individual's sexual partner returned from an affected area and developed clinical symptoms. About $80 \%$ of people infected with the Zika virus do not develop any clinical symptoms. Nevertheless, of those who do experience clinical symptoms, they are usually mild and include fever, arthralgia, conjunctivitis (red eyes) or a rash. Other clinical symptoms can include headache and myalgia.

The incubation period is likely to be a few days to a week. The illness is usually mild with symptoms lasting for several days to a week. The virus usually remains in the blood for a few days, but it can be identified longer in some individuals. Cases with severe disease requiring hospital admission is uncommon. There are at least 72 persons who have been diagnosed with the Zika virus infection or disease in the United States since the latest outbreak began. At least four pregnant women have been confirmed the diagnosis of Zika virus infection. All of the infected cases are believed to have been travel related except for one that was transmitted through sexual contact from an infected traveler to their partner that mentioned before. The disease has historically occurred in Africa, Southeast Asia, and islands in the Pacific Ocean. Zika virus disease is considered an emerging infectious disease with potential to spread to new areas. In May 2015, the Pan American Health Organization (PAHO) issued an alert regarding the first confirmed Zika virus infections in northeastern Brazil. The virus has since spread throughout much of the South America, Central America, and the Carribean.

They had more evidence that links the Zika virus to a dangerous birth defect namely" microcephaly". The genetic material of the Zika
Volume 3 Issue 2 - 2016

\author{
Attapon Cheepsattayakorn, ${ }^{1,2,3}$ Ruangrong \\ Cheepsattayakorn ${ }^{4}$ \\ 'Senior Editor, Journal of Human Virology and Retrovirology, \\ USA \\ ${ }^{2}$ Oth Zonal Tuberculosis and Chest Disease Center,Thailand \\ 35th Office of Disease Prevention and Control, Ratchaburi, \\ Department of Disease Control, Ministry of Public Health, \\ Thailand \\ ${ }^{4}$ Department of Pathology, Faculty of Medicine, Chiang Mai \\ University, Thailand
}

Correspondence: Attapon Cheepsattayakorn, I0th Zonal Tuberculosis and Chest Disease Center, 143 Sridornchai Road, Changklan Muang, Chiang Mai, 50100 Thailand, Tel 6653 I40767, 6653 276364, Fax 6653 140773, 6653 273590, Email attapon1958@gmail.com, attaponche@yahoo.com

Received: February 16, 2016| Published: February 17, 2016

virus was identified in brain tissue from two infants with microcephaly who had finally died. The United States Centers for Disease Control and Prevention recommends that pregnant woman consider postponing travel to any area where the Zika virus transmission is ongoing. Despite these relatively mild clinical symptoms, several health officials are investigating a possible association between Zika virus infections in pregnant women and certain birth defects. The diagnosis of Zika virus infection is performed through serologic and molecular testing that includes reverse transcriptase-polymerase chain reaction (RT-PCR) for viral RNA, and immunoglobulin (Ig) M ELISA and plaque reduction neutralization test (PRNT) for Zika virus antibodies. Individuals prevent Zika virus infection by avoiding mosquito bites. Currently, there is no vaccine or medications are available to prevent or treat Zika virus infection.

\section{Acknowledgments}

None.

\section{Conflicts of interest}

None. 